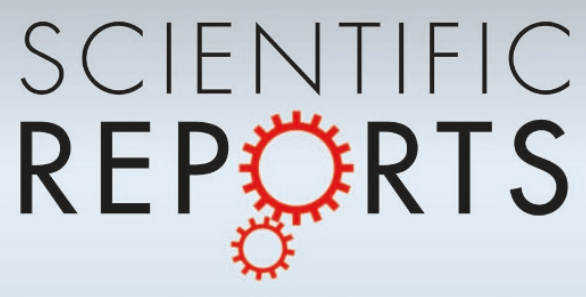

OPEN

SUBJECT AREAS:

MECHANICAL

PROPERTIES

SURFACES, INTERFACES AND THIN FILMS

Received

17 October 2014

Accepted

2 February 2015

Published

3 March 2015

Correspondence and requests for materials should be addressed to K.N.S. (kiran.solanki@ asu.edu)

\section{Structural stability and energetics of grain boundary triple junctions in face centered cubic materials}

\author{
I. Adlakha \& K. N. Solanki
}

School for Engineering of Matter, Transport, and Energy, Arizona State University, Tempe, AZ.

We present a systematic study to elucidate the role of triple junctions (TJs) and their constituent grain boundaries on the structural stability of nanocrystalline materials. Using atomistic simulations along with the nudge elastic band calculations, we explored the atomic structural and thermodynamic properties of TJs in three different fcc materials. We found that the magnitude of excess energy at a TJ was directly related to the atomic density of the metal. Further, the vacancy binding and migration energetics in the vicinity of the TJ were examined as they play a crucial role in the structural stability of NC materials. The resolved line tension which takes into account the stress buildup at the TJ was found to be a good measure in predicting the vacancy binding tendency near the TJ. The activation energy for vacancy migration along the TJ was directly correlated with the measured excess energy. Finally, we show that the resistance for vacancy diffusion increased for TJs with larger excess stored energy and the defect mobility at some TJs is slower than their constituent GBs. Hence, our results have general implications on the diffusional process in NC materials and provide new insight into stabilizing NC materials with tailored TJs.

$\mathrm{N}$ anocrystalline (NC) materials (mean grain size, $d<100 \mathrm{~nm}$ ) often have enhanced mechanical properties compared to coarse-grained materials $(d>1 \mu \mathrm{m})$. Therefore, $\mathrm{NC}$ alloys are very attractive for multiple engineering applications including load-bearing structures ${ }^{1}$. The challenge with broader applicability of NC materials has been the stability of the non-equilibrium microstructure during processing and deformation. The structural stability, mechanical behavior, and fracture of NC materials is often driven by grain boundaries (GBs, planar defects), triple junctions (TJs, line defects), and their underlying structures ${ }^{2,3}$. Hence a fundamental understanding of the relationship between the linear/planar defect structures and the associated properties is important in the development of interface-dominant materials, especially in the case of NC materials where TJs constitute a large volume fraction ${ }^{3}$. Further, the role of $\mathrm{TJ}$ atomic structure on mass transport or diffusional processes, which directly controls the stability of NC materials, is a critical open question.

In NC materials, the grain growth behavior is typically characterized with the help of classical (2D) approaches such as the original von Neumann-Mullins relation (refer to further detail in Mullins) ${ }^{4}$. However, such a model provides an inadequate description of the grain growth behavior in NC materials as it ignores TJ contributions on the evolution of the grain growth behavior. In fact, Czubyako et al. ${ }^{5}$ experimentally showed that a low TJ mobility can induce a significant drag effect on the grain growth and subsequently proposed a modified von NeumannMullins relationship that, to some extent, captures the interplay between the GB and TJ kinetics on the grain growth behavior ${ }^{5}$. Nevertheless, such models are derived based on geometrically constrained TJs and do not account for the varying TJ structures to accurately capture the intrinsic role of TJs on the grain growth behavior of $\mathrm{NC}$ materials $s^{6-9}$. On the other hand, a number of groups have linked the structural stability of TJs to thermodynamic variables, such as the excess free energy, the resolved line tension, and the resolved line force of $\mathrm{TJ}^{6,10,11}$. For example, Gottstein et al. have shown that the excess Gibbs energy at the TJ can serve as a measure of the TJ mobility ${ }^{6}$. However, there is a lack of systematic investigations exploring various thermodynamic properties of TJs formed by commonly observed $\mathrm{GBs}^{12-16}$ and their links to the structural stability of NC materials, i.e., defect migrations.

In this work, for the first time, we present a systematic investigation using molecular statics along with the nudge elastic band (NEB) method ${ }^{17}$ to quantify the intrinsic properties for a range of special TJs (these TJs have been shown to occur frequently ${ }^{12,13,16}$ ) in $\mathrm{Al}, \mathrm{Cu}$, and $\mathrm{Ni}$ (see Table S1). The choice of materials studied in this manuscript highlights a large variation in the elastic and plastic anisotropies. The elastic anisotropy ratio, 
$A=\frac{2 C_{44}}{C_{11}-C_{12}}$, for $\mathrm{Al}, \mathrm{Ni}$, and $\mathrm{Cu}$ is $1.16,2.5$, and 3.19, respectively, indicating that the $\mathrm{Al}$ is highly isotropic as compared to $\mathrm{Ni}$ and $\mathrm{Cu}$. Similarly, the ratio between the stable and unstable stacking fault energies $\left(\frac{\gamma_{s f}}{\gamma_{u s f}}\right)$ can be used to comprehended the plastic behavior of these materials, i.e., the ratio describes the competition between a full versus partial dislocation ${ }^{18}$. For instance, $\mathrm{Al}$ has the highest ratio between the stable and unstable stacking fault energies (0.83) followed by $\mathrm{Ni}(0.34)$ and $\mathrm{Cu}(0.28)^{19-21}$. Thus, these metals show a wide variation in critical elastic and deformation properties providing a broad platform to analyze the general behavior of TJs. For example, the role of TJ atomic structure on the vacancy binding and migration energetics was examined in the present manuscript as the vacancies can serve as an effective means for relieving the accumulated strain energy at the $\mathrm{TJ}^{22}$. However, when concentration of vacancies in the bulk exceeds the equilibrium concentration, it leads to an increase in the Gibbs free energy and produces a reactive thermodynamic force. This thermodynamic force provides resistance to the TJ motion during grain growth.

Finally, the relationship between the measured intrinsic properties and the structural stability was examined in detail. The vacancy binding energetics and the migration behavior is directly related to the structural stability of TJs ${ }^{22}$. Hence, it is critical to understand the intrinsic role between TJ thermodynamic properties and vacancy binding/migration characteristics. Therefore, our results have general implications on the diffusional/transport process in NC materials and provide new insight into stabilizing NC materials with tailored TJs through patterning ${ }^{23}$ or GB engineering ${ }^{24}$.

\section{Results}

Triple junction atomic structure. First, we focus on characterizing the TJ atomic structures by measuring the hydrostatic stress field and the net change in atomic volume due to the formation of TJs. These structural quantities provide a basis for measuring the $\mathrm{TJ}$ intrinsic properties, such as the excess energy, the resolved line tension and the resolved line force. For instance, the hydrostatic stress field is further used to compute the resolved line tension acting at the TJ, and the measured change in volume is directly proportional to the TJ misfit strain. Figure 1 shows the atomic hydrostatic stress field for a few $(\Sigma 3-\Sigma 3-\Sigma 9, \Sigma 3-\Sigma 9-\Sigma 27, \Sigma 3-\Sigma 11-\Sigma 33$, and $\Sigma 3-\Sigma 27-\Sigma 81)$ TJs in Al highlighting the atomic arrangement due to GBs intersecting at the TJ. Details related to the construction of various TJs are presented in the methods section. The hydrostatic stress accumulated near the $\Sigma 3-\Sigma 3-\Sigma 9$ TJ (Figure 1a) was higher when compared to the stress along the GBs. This clearly highlights that in the case of the $\Sigma 3-\Sigma 3$ $\Sigma 9$, the TJ has a greater influence on the stress accumulation. On the other hand, in the case of the $\Sigma 3-\Sigma 9-\Sigma 27$, the constituent GBs along with the terminating GB structures at the TJ have a large effect on the accumulated stress. In the case of the $\Sigma 3-\Sigma 27-\Sigma 81$ (Figure 1d), an intrinsic stacking fault (ISF) was formed in the vicinity of the TJ. The localization of large stresses along the terminated GB structures can be attributed to the formation of the ISF, and further, the ISF can act as another defect sink.

The net change in volume due to the formation of the TJ was also computed using the following relation to comprehend the misfit strain induced during the formation of a TJ.

$$
\begin{aligned}
& \Delta V_{T J}=\pi r_{T J}^{2}-\frac{n \omega_{\text {bulk }}}{r_{T J}}-\sum_{i=1}^{3} l_{x}^{i} \Delta V_{G B}^{i} \\
& r_{T J}=\max \left(l_{x}^{i}\right)
\end{aligned}
$$

Here, $r_{T J}$ is the radius for the TJ for calculating net change in volume, $\mathrm{n}$ is the number of atoms lying with a distance of $r_{T J}$ from the TJ, $\omega_{\text {bulk }}$ is the atomic volume in a pristine fcc lattice at $0 \mathrm{~K}$, and $\Delta V_{G B}^{i}$ is the volume expansion along the GB plane per unit of the GB area for the $i^{\text {th }}$ interface, and $l_{x}^{i}$ is the length of the unit vector tangential to the GB plane. The net change in normalized volume for all the metals follows no clear trend except that TJs with high $\Sigma$ GBs undergo greater volumetric expansion in contrast to other TJs, see Figure 2a (also refer to Tables S2, S3, and S4). This suggests that the high $\Sigma$ GBs would form a more diffused TJ. A similar trend was reported for TJs in Si using atomistic simulations ${ }^{25}$. In general, the volume change for $\mathrm{Al}$ TJs was found to be greater than those of the $\mathrm{Cu}$ and Ni. This variation is directly related to the differences in the lattice parameters of these metals $\left(a_{A l}>a_{C u}>a_{N i}\right)$.

Characterizing triple junction thermodynamic properties. The total excess energy due to the formation of the TJ was computed with respect to the energy of the pristine fcc lattice. The excess energy due to the formation of TJs was found using the following relation:

$$
\Delta E_{T J}=\left(E_{T J}-n E_{c o h}-\sum_{i=1}^{3} E_{G B}^{i} l_{x}^{i} l_{z}\right) / l_{z}
$$

where $E_{T J}$ is the energy of the defected structure with TJ and GB interfaces, $E_{c o h}$ is the cohesive energy of each atom at $0 \mathrm{~K}$ in a pristine fcc lattice, and $E_{G B}^{i}$ is the GB formation energy per unit area for the $i^{\text {th }}$ interface. The net excess energy was normalized by the line length $\left(l_{z}\right)$ of the TJ. These results were found by the summation of atomic energies within a distance of $19 \mathrm{~nm}$ from the TJ. The trends for normalized excess energy for all the metals studied were found to be comparable (Figure 2a). For a few TJs studied in the open literature using atomistic methods ${ }^{12,25,26}$ and experimental work ${ }^{27}$, the excess energy computed here is in close agreement. The excess energy for TJs in Al formed by low energy GB interfaces was found to be higher when compared to the TJs with high energy interfaces. A similar trend was reported for TJs in Si using atomistic simulations ${ }^{25}$.

Next, we evaluate the resolved line tension which has been shown as a driving force for the TJ mobility during microstructural evolu$\operatorname{tion}^{5,6,8-10}$. The resolved line tension was computed using the relationship described in the method section. The variation in the resolved line tension trend (Figure $2 \mathrm{~b}$ ) correlates with the elastic anisotropy of metals studied, i.e., $\mathrm{Cu}\left(A_{C u}=3.19\right)$ has the highest variation in the resolved line tension followed by $\mathrm{Ni}\left(A_{N i}=2.5\right)$ and $\mathrm{Al}\left(A_{A l}=1.16\right)$. The relative stability of TJs based on the excess energy and the resolved line tension for the $\Sigma 3^{n}-\Sigma 3-\Sigma 3^{n-1}$ TJs was found to be lower than that of $\Sigma 3^{n}-\Sigma 3-\Sigma 3^{n+1}$ TJs (Figures $2 \mathrm{a}$ and $2 \mathrm{~b}$ ). Similar conclusions have been drawn in previous studies on TJs in Ni and other fcc materials ${ }^{28-30}$. The resolved line force acting at the TJ was computed with the help of the excess energies and the spatial arrangement of the intersecting GBs at the TJ. There was no clear trend observed between the resolved line force for the three materials and other intrinsic quantities (Table S2-4).

Correlating the triple junction thermodynamic properties to vacancy binding energetics. The vacancy binding energy in the vicinity of the TJ $(\leq 4 \mathrm{~nm})$ was investigated, see Figure 3 . The vacancy binding energy $\left(E_{b}\right)$ is essentially the extra energy required to form a defect at a specific site near the defect region (in this case the TJ, $E_{f}^{T J}$ ) in comparison to the bulk formation energy of the defect $\left(E_{b}^{T J}=E_{f}^{b u l k}-E_{f}^{T J}\right.$, where $E_{f}^{b u l k}$ for $\mathrm{Al}, \mathrm{Cu}$, and $\mathrm{Ni}$ was $0.71,1.29$, and $1.64 \mathrm{eV}$ respectively, refer to Refs. 31, 32 and Supplementary material). The effect of TJs on the binding tendency for atomic sites within the grain was found to be negligible, i.e., the atoms further away from the TJs are colored white $(0 \mathrm{eV}$ vacancy binding energy), indicating that there is no energy difference over the bulk lattice binding energy (see Figure 3). There is a clear difference in the numbers of preferred binding sites $\left(E_{b}^{T J}>0\right)$ in the vicinity of the TJ between the $\Sigma 3-\Sigma 3-\Sigma 9$ and $\Sigma 3-\Sigma 9-\Sigma 27$ TJs (Figure $3 \mathrm{a}$ and $3 \mathrm{~b}$ ). The $\Sigma 3-\Sigma 9-\Sigma 27 \mathrm{TJ}$ has preferred vacancy sites concentrated in a very 


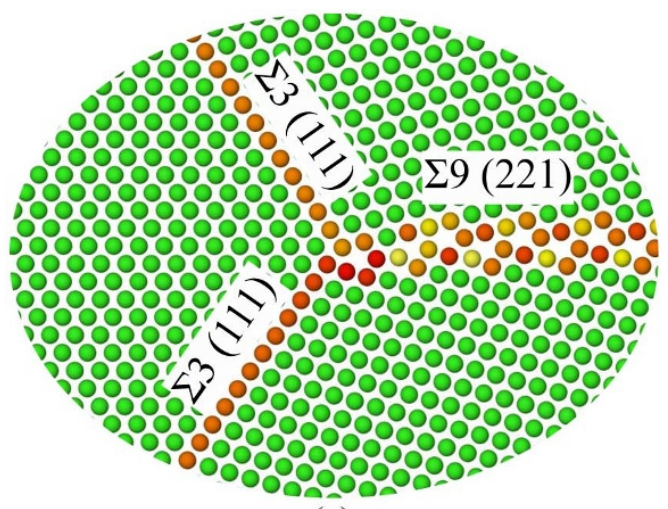

(a)

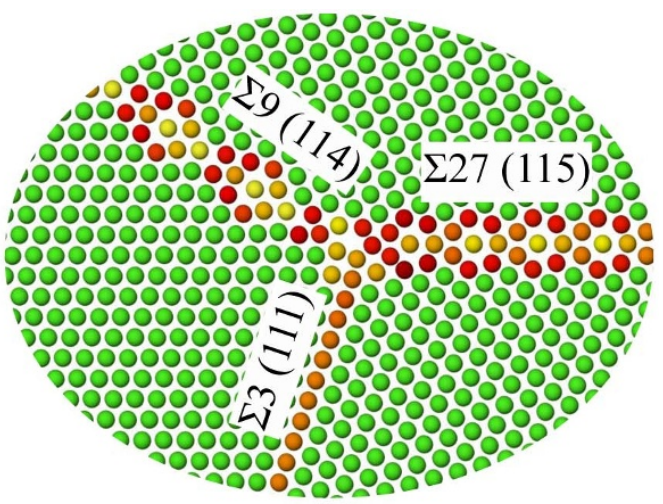

(b)

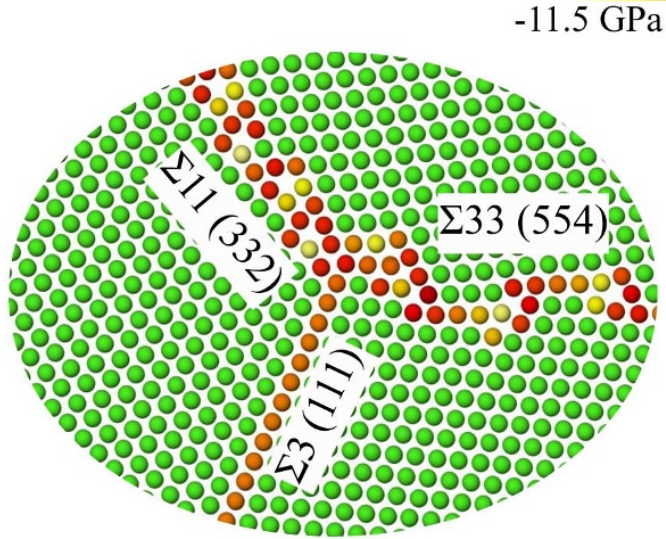

(c)

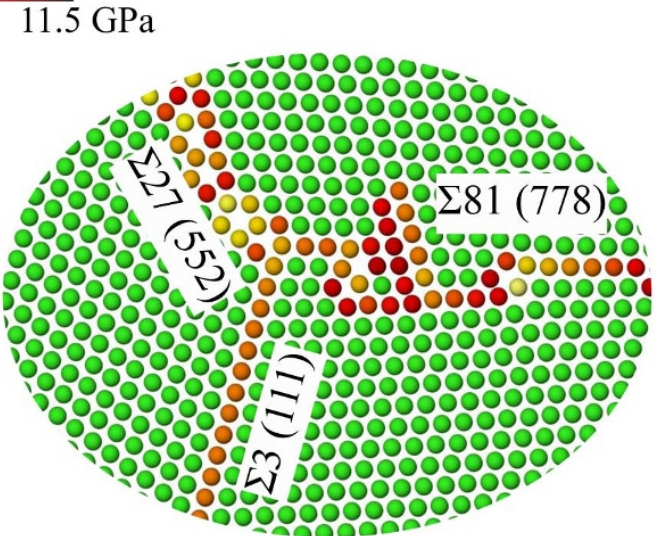

(d)

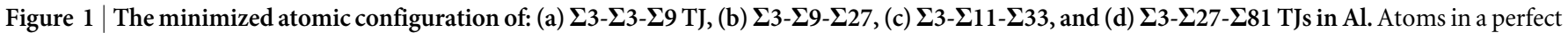
fcc lattice are depicted as green while the defected atoms along the GB and the TJ are colored according to the variation in hydrostatic stress.

small region around the TJ ( 3 A radially) indicating the extent of the TJ elastic strain field. On the other hand, the $\Sigma 3-\Sigma 19-\Sigma 57$ and $\Sigma 3-$ $\Sigma 27-\Sigma 81$ TJs have more stable vacancy sites $\left(E_{b}^{T J}>0\right)$ away from the TJ along the constituent GBs and along the ISF formed during the minimization of the TJ (Figures $3 \mathrm{c}$ and $3 \mathrm{~d}$ ).

Furthermore, the role of constituent GBs and the TJ on vacancy binding was investigated with the help of the mean binding energy as a function of radial distance from the TJ (Figure 4). The vacancy binding energy for a majority of TJs converged to the bulk vacancy formation energy at a length of approximately $2 \mathrm{~nm}$ from the TJ. In all three fcc metals studied, the vacancy binding energy was maximum (i.e. $<10 \AA$ radially) for the TJs that had the maximum resolved line tension (Figure 2b). Nonetheless, there were a few exceptions such as the $\Sigma 3-\Sigma 19-\Sigma 57 \mathrm{Al}$ and $\Sigma 3-\Sigma 3-\Sigma 9 \mathrm{Cu}$ that also showed lower binding energy near the TJ. The reason for the observed lower binding energy for the $\Sigma 3-\Sigma 19-\Sigma 57 \mathrm{Al}$ was due to the greater influence of GBs over the TJ.

Vacancy migration along the triple junction line. Finally, using the NEB method, the role of TJ local structure on the kinetics of vacancies, i.e., migration energy within the TJ plane (Figure 5a) was characterized. The vacancy migration energy for both $\mathrm{Al}$ and $\mathrm{Cu}$ follows a similar trend. In general, the vacancy migration energy along the TJs in Ni was clearly greater than its counterparts in $\mathrm{Al}$ and $\mathrm{Cu}$. The high vacancy migration energy in Ni TJs can be attributed to the availability of a large number of stable sites for vacancy binding

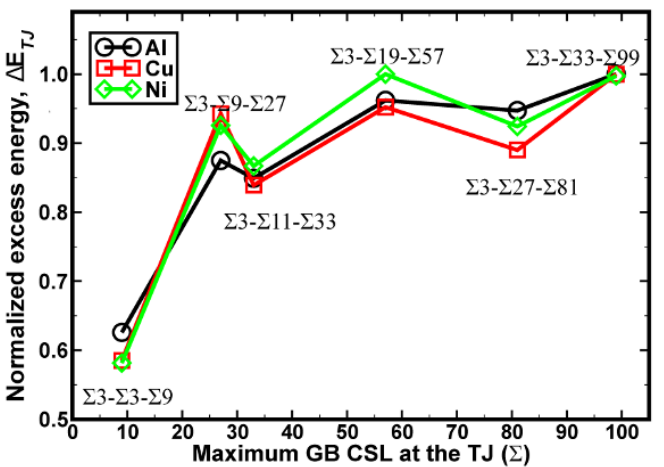

(a)

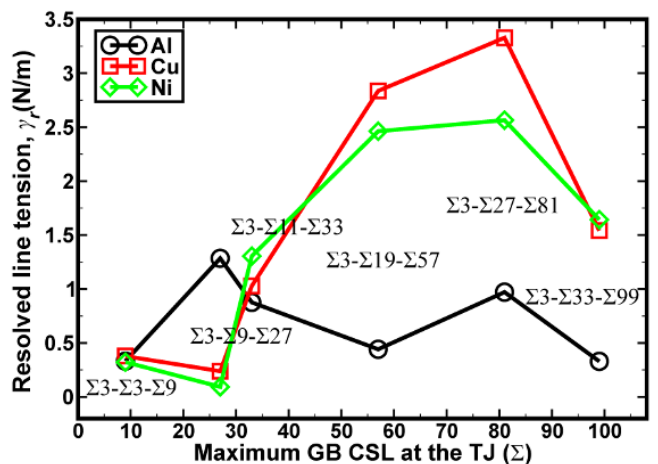

(b)

Figure $2 \mid$ (a) The normalized excess energy of TJs in $\mathrm{Al}, \mathrm{Cu}$, and $\mathrm{Ni}$. A clear trend can be observed indicating that the relative structural stability of the TJ was similar regardless of the chosen material type. (b) The resolved line tension acting along the $\mathrm{TJ}$ for $\mathrm{Al}, \mathrm{Cu}$, and $\mathrm{Ni}$. 


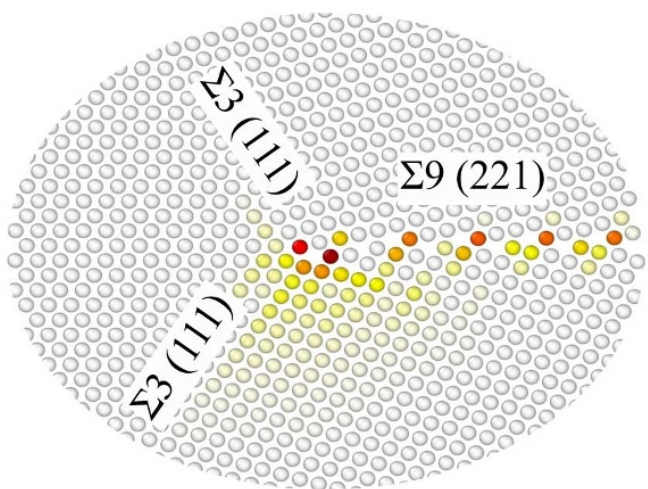

(a)

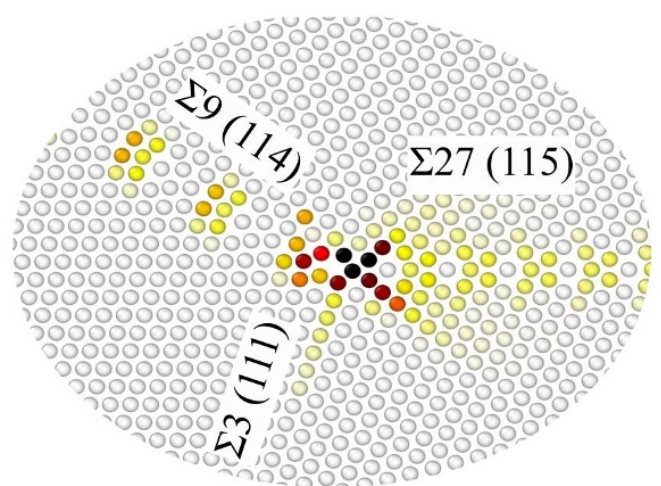

(b)

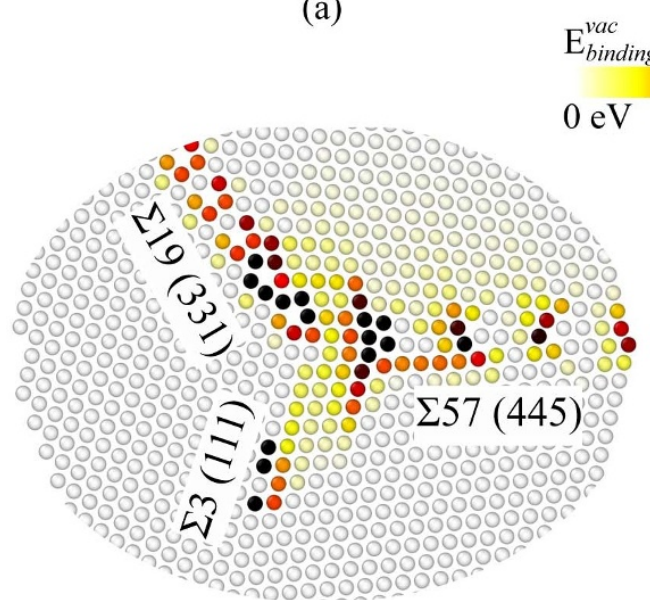

(c)

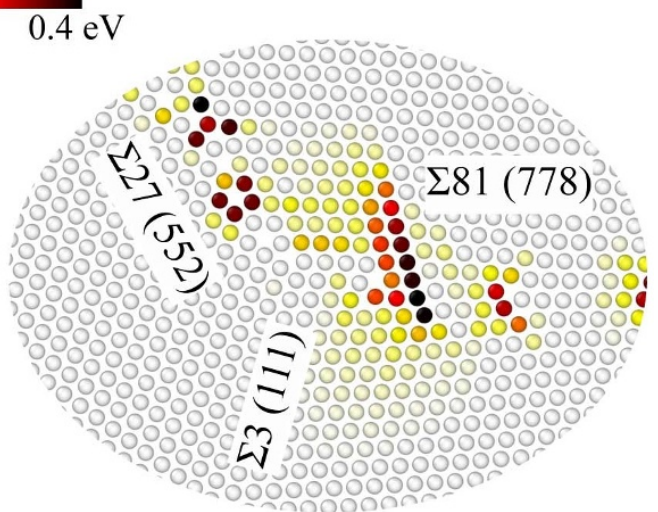

(d)

Figure $3 \mid$ The atomic variation in vacancy binding energy near (a) $\Sigma 3-\Sigma 3-\Sigma 9$, (b) $\Sigma 3-\Sigma 9-\Sigma 27$, (c) $\Sigma 3-\Sigma 19-\Sigma 57$, and (d) $\Sigma 3-\Sigma 27-\Sigma 81$ TJs in Al. The atoms were colored based on the vacancy binding energy at each site. White atoms correspond to the bulk binding energy $(\sim 0 \mathrm{eV})$ and black atoms represent the maximum vacancy binding energy of $0.4 \mathrm{eV}$.

near the TJ, as shown in Figure 4c. The $\Sigma 3-\Sigma 27-\Sigma 81 \mathrm{TJ}$ in Ni was found to have migration energy of $2 \mathrm{eV}$ (Figures $5 \mathrm{a}-\mathrm{b}$ ). The high migration energy can be explained with the help of the binding energy for the TJ (Figure 4c). The presence of extremely stable binding $(\sim 1.2 \mathrm{eV})$ sites for vacancies around the $\Sigma 3-\Sigma 27-\Sigma 81 \mathrm{TJ}$ (Figure $4 \mathrm{c}$ ) require far more energy to enforce vacancy migration (Figure 5b), clearly indicating a drag effect on the defect migration.

The effect of the interplay between a GB and the TJ on the grain growth has been discussed in previous work ${ }^{5,9}$. For example, it has been shown that below a certain critical grain size, the immobile TJs can create a drag effect to the microstructural growth. Here, we characterize the mass transport properties of the TJs by quantifying the activation energy for diffusion, i.e., the sum of vacancy formation and migration energies for various TJs (refer to Table S5).

$$
Q=E_{f}^{T J}+E_{m i g}^{T J}
$$

where $E_{m i g}^{T J}$ is the vacancy migration energy in the vicinity of the TJ.

The normalized activation energy for vacancy diffusion was plotted against the normalized excess energy due to formation of the TJs (Figure 6). In the case of $\mathrm{Cu}$ TJs, there was a strong correlation between the activation energy barrier and the excess energy. Conversely, activation energy was weakly correlated to the excess energy for TJs in Al. The activation energy for TJs in Ni was found to be insensitive to the excess energy of the TJ.

\section{Discussion}

As mentioned earlier, the microstructural stability is critical for broader applicability of NC materials. Therefore, it is critical to evaluate the role of TJs and their constituent GBs on the structural stability. In this regard, the effect of the interplay between the TJs and their constituent GBs on the physical properties of NC materials has, so far, been myopically categorized into: a) the TJ behavior/properties can be entirely described by their constituent GBs; b) the formation of a TJ due to termination of the interface causes a change in the behavior of one or more constituent GBs; and c) the properties of TJs are independent of their constituent $\mathrm{GBs}^{33}$. Thus, to account for this interplay, for the first time, the microstructural stability of various TJs with frequently observed GBs in fcc metals ( $\mathrm{Al}, \mathrm{Ni}$, and $\mathrm{Cu}$ ) have now been examined.

The quantification of TJ mobility in NC materials while incorporating the atomistic scale details is a challenging prospect with current experimental and modelling techniques. Further, the measurement of intrinsic structural and thermodynamic quantities of the TJ at the atomistic scale is critical as these quantities are thermodynamically related to the mobility of the $\mathrm{T} \mathrm{J}^{6,10,11}$. The hydrostatic stress field and the net volume change can be used to measure the degree of variability in the TJ local structural arrangement, which influences the mechanical behavior of NC materials. For example, the local TJ misfit strain is a function of the net volume change during the formation of the TJ, which can significantly alter the vacancy binding energetics (Figure 3). Further, the net volume change is directly related to the lattice parameter of the metals (Table S2-4), i.e., the largest volumetric changes were observed for $\mathrm{Al}$ followed by $\mathrm{Cu}$ and $\mathrm{Ni}$ TJs, respectively. Similarly, the variation in the atomic scale hydrostatic stress field of the TJ (Figure 1) indicates that the stress buildup at the TJ is not correlated with the GB coincident site lattice value (CSL) or GB energy. In other words, a TJ formed by intersecting GBs with high CSL values or GB energies can have a small hydrostatic stress distribution. These measured local structural prop- 


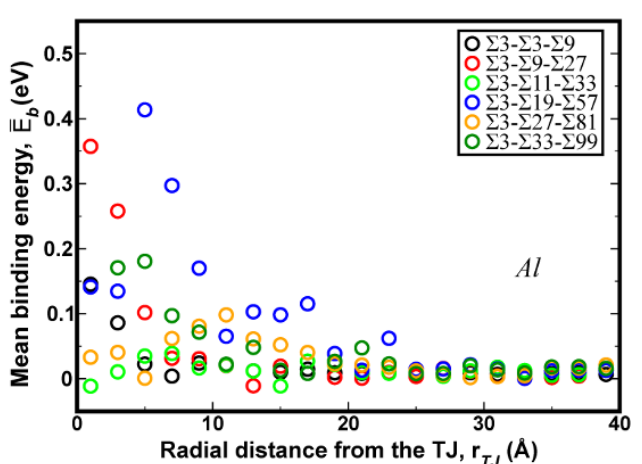

(a)

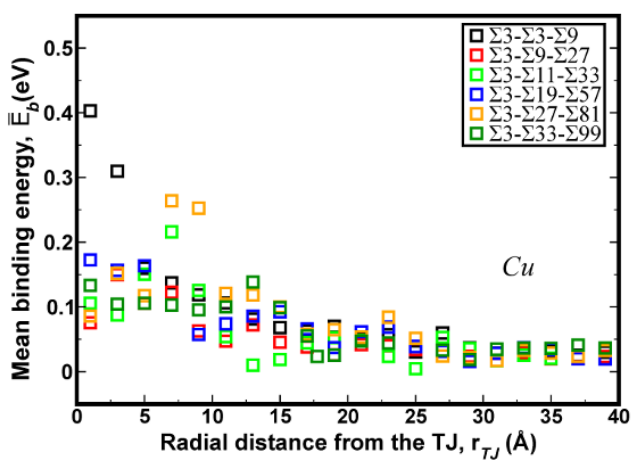

(b)

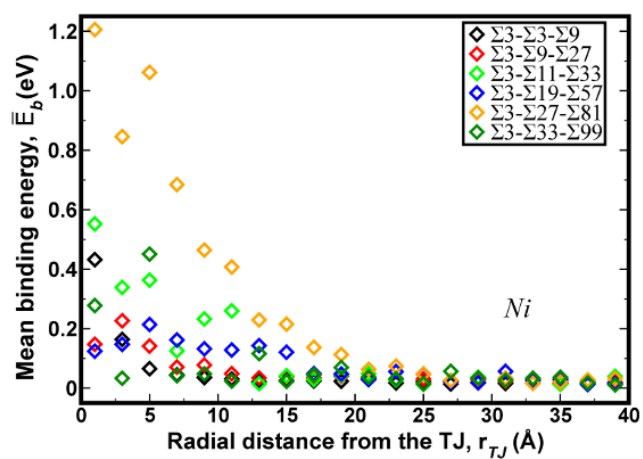

(c)

Figure $4 \mid$ The mean vacancy binding energy as a function of radial distance from the $\mathrm{TJ}$ for (a) $\mathrm{Al}$, (b) $\mathrm{Cu}$, and (c) Ni. A total of 20 concentric bins were defined $\left(r_{i-1} \leq r \leq r_{i}\right)$ up to $4 \mathrm{~nm}$ from the TJ. In the cases of $\mathrm{Al}$ and $\mathrm{Ni}$, the maximum binding energy was observed for TJs with the highest resolved line tension clearly suggesting that higher energy TJs were favorable sites for the vacancy binding.

erties, as highlighted here, are related to various thermodynamic quantities of the TJ, which significantly influence the stability of NC materials. For instance, the high hydrostatic stress distribution around the TJ can be relieved through the transport of vacancies. The magnitude of resolved line tension, which is a function of the hydrostatic stress field of the TJ, is related to that of the maximum vacancy binding tendency at the TJ (Figure 4). That is the TJs with higher resolved line tensions exhibit a large number of stable sites for the vacancy binding in the immediate vicinity of the TJ (Figure 3 ). Therefore, TJs with a higher resolved line tension can induce a significant drag on the mobility of the TJ with respect to its constituent $\mathrm{GBs}^{5}$. The interplay between the GB and TJ dominates the vacancy

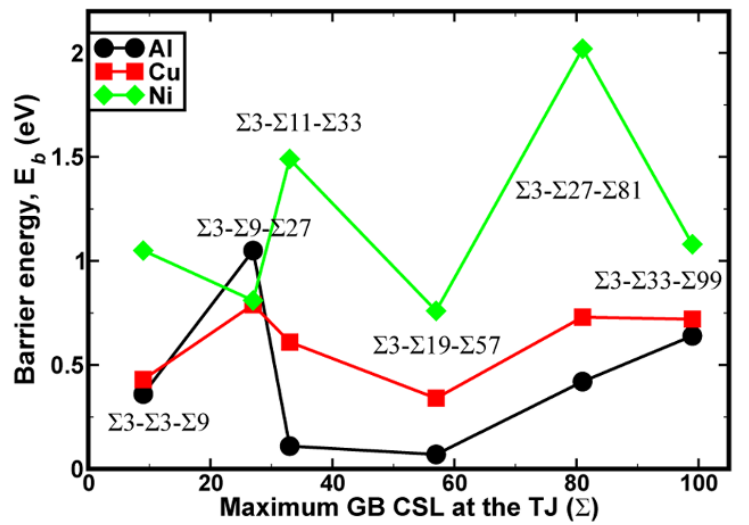

(a) binding behavior. For instance, in a $\Sigma 3-\Sigma 3-\Sigma 9 \mathrm{Al}$ TJ, the stable sites for binding were clustered near the TJ suggesting a strong influence of the TJ, Figure $3 \mathrm{a}$. On the other hand, in the case of the $\Sigma 3-\Sigma 19-\Sigma 57$ TJ, the stable sites were spread along the GB, implying that the GB structure and the relative position of the TJ to the GB structure are critical Figure 3c.

The flux of vacancies from the TJ to the bulk offers a faster method for relieving stored elastic energy results in the subsequent rearrangement of the GB network ${ }^{22}$. Hence, the excess energy measurement of the $\mathrm{TJ}$ is also intuitively appealing since it takes into account the $\mathrm{TJ}$ atomic structural arrangement and it is related to the TJ mobility. Moreover, as evident from Figure 2a, the magnitude of excess energy

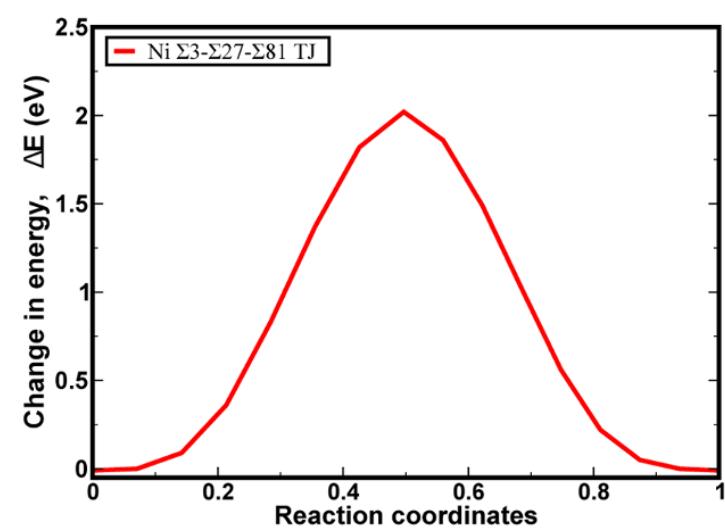

(b)

Figure $5 \mid$ (a) The vacancy migration energies for $\mathrm{Al}, \mathrm{Cu}$, and $\mathrm{Ni}$ along the TJ. (b) The change in energy as the vacancy migrates in the Ni $\Sigma 3-\Sigma 27-\Sigma 81 \mathrm{TJ}$. In the case of $\mathrm{Al}$ and $\mathrm{Cu}$, the migration energies trend correlates with the excess energies. In general, the vacancy migration energies of Ni TJs were much higher than that of $\mathrm{Al}$ and $\mathrm{Cu}$ TJs. The possible explanation for this trend can be attributed to the higher vacancy binding observed within the TJs plane in $\mathrm{Ni}$. 


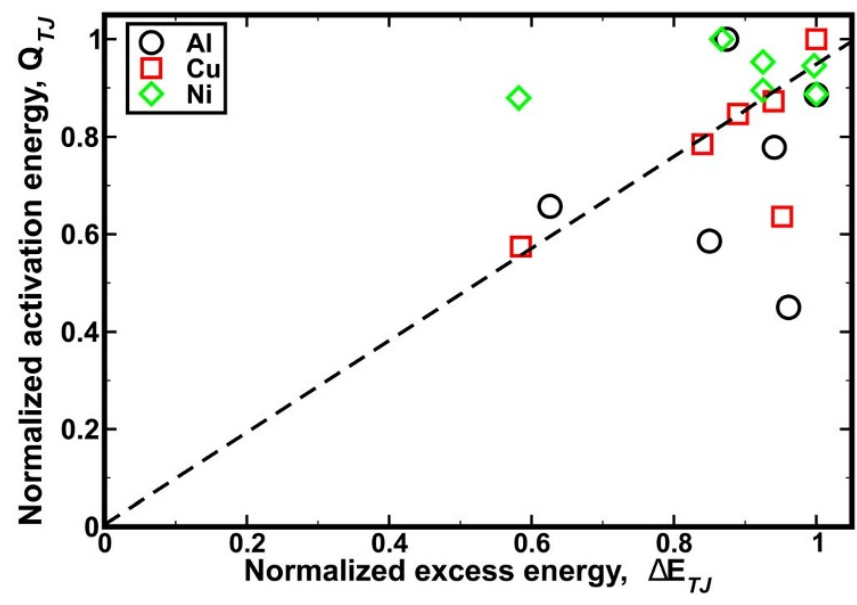

Figure 6 | The normalized activation energy for diffusion in $\mathrm{Al}, \mathrm{Cu}$, and Ni TJs was plotted as a function of normalized excess energy. The extent of correlation between these thermodynamic quantities for various metals can be summarized as $\mathrm{Cu} \gg \mathrm{Al}>\mathrm{Ni}$ (Ni was largely insensitive to the change in excess energy stored at the TJ).

for the TJs $\left(\Delta E_{T J}^{N i}>\Delta E_{T J}^{C u}>\Delta E_{T J}^{A l}\right)$ are inversely related to the lattice parameter of the material investigated here $\left(a_{A l}>a_{C u}>a_{N i}\right)$. In other words, a higher atomic density of the metal results in a lower dissipation of the excess energy during formation of the TJ. The excess stored energy during the formation of the TJ can be a realistic measure for predicting the structural stability of the GB network, see Figure 6. For instance, for $\mathrm{Cu}$ TJs, there is a strong correlation between the activation energy barrier and the excess energy, i.e., an increase in the excess energy results in a significant drag effect on the $\mathrm{TJ}$ mobility. In the case of $\mathrm{Ni}$, the trends between the excess energy and activation energy are not correlated because of the high excess energies for all Ni TJs. Nonetheless, these findings are in agreement with previous works on the drag effect created by TJs during grain growth $^{7,9,22,34}$.

In summary, for the first time, various intrinsic quantities of the TJ at the atomic scale were systematically quantified. Hence, this study provides generalized insights regarding the structural stability of NC materials. Moreover, these new atomistic perspectives provide a physical basis for recognizing the role between the TJ and vacancy transport in fcc materials. Since, both kinetic and thermodynamic properties of defects are very different at different triple junctions in different materials. Further, the defect mobility at some triple junctions can be slower than their constituent GBs. This is significant for applications where extreme environment damage generates lattice defects and TJs act as sinks for both vacancies and interstitial atoms. Further, our results have general implications on the diffusional/ transport process in NC materials and provide new insight into stabilizing NC materials with tailored TJs through patterning ${ }^{23}$ and/or GB engineering ${ }^{24}$.

\section{Methods}

Molecular statics simulations using LAMMPS ${ }^{35}$ were employed in this work to investigate the structural stability of TJs. The atomic interactions in this work were described using the embedded atom method (EAM) potential for $\mathrm{Cu}, \mathrm{Ni}$, and $\mathrm{Al}^{19-21}$. These EAM potentials were parameterized using an extensive database of energies and configurations from density functional theory calculations and have been used to accurately define different material behaviors such as surface energies, generalized stacking fault energies, etc. (e.g., Refs. 19-21). In this work, TJs were constructed using $<110>$ symmetric tilt grain boundaries (GBs), see Table S1. These GBs were characterized using the structural unit method ${ }^{36,37}$. As with past work ${ }^{31,32,38,39}$, an atom deletion criterion, multiple initial configurations, and various in-plane rigid body translations were utilized to accurately obtain an optimal minimum energy GB structure via the conjugate gradient energy minimization process. The energy and force minimization convergence criterion was 1e-25. Circular wedges with a radius of $20 \mathrm{~nm}$ from TJ were then cut from the GBs along the stitch plane, i.e. $\{001\} /\{011\}$ planes, see Figure $7^{15,40}$.

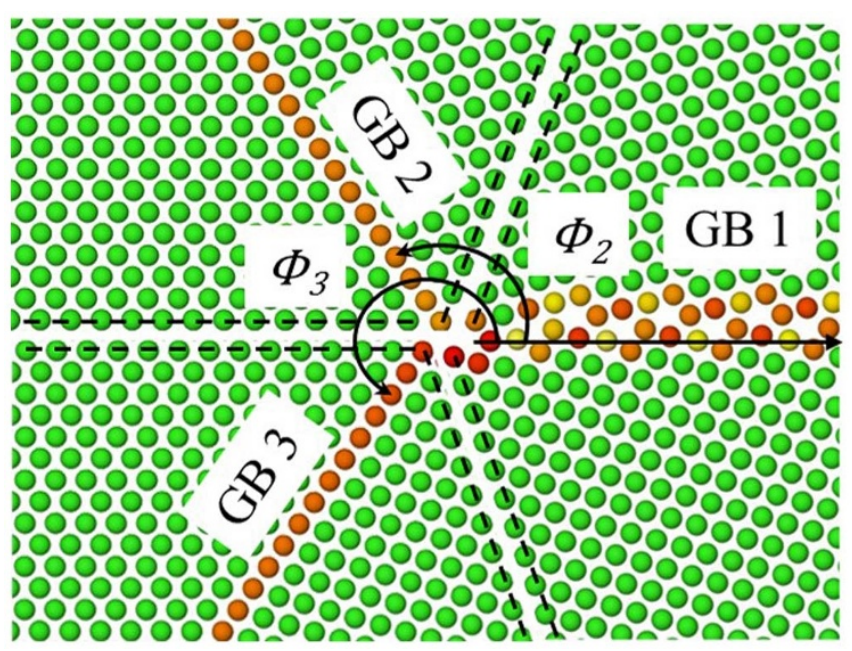

Figure 7 | Schematic showing construction of a GB TJ configuration. The GBs were minimized separately and a wedge was carved out along the $\{001\} /\{011\}$ plane represented by dashed lines.

Subsequently, GBs labelled 2 and 3 were rotated by $\phi_{2}$ and $\phi_{3}$ about the GB tilt axis as shown in Figure 7. Lastly, the three wedges were brought together, the overlapping atoms were removed, and the energy minimization was carried out at $0 \mathrm{~K}$. According to the Herring's relation the TJ attempts to minimize the resolved surface tension during formation. The resolved line tension acting at the TJ was approximated at the atomic scale using the following relation:

$$
\gamma_{r}=\sum_{i=1}^{3} \gamma_{i} l_{x}^{i}
$$

The surface tension for the intersecting GBs $\left(\gamma_{i}\right)$ was defined by averaging normal and tangential stresses acting over a region of $\pm 20 \AA$ normal to the interfaces and up to $19 \mathrm{~nm}$ from the TJ. The resolved line force was another quantity used to understand the energetics of TJs. This was defined as:

$$
\begin{aligned}
& F_{i}=\Delta E_{i} l_{x}^{i} \\
& F_{T J}=\sqrt{\left(\sum_{i=1}^{3} F_{i} \cos \phi_{i}\right)^{2}+\left(\sum_{i=1}^{3} F_{i} \sin \phi_{i}\right)^{2}}
\end{aligned}
$$

The excess energy per unit GB area $\left(\Delta \mathrm{E}_{\mathrm{i}}\right)$ subsequent to the formation of the TJ was defined over a region of $\pm 20 \AA$ normal to the GB. The line force for all the GBs was resolved at the $\mathrm{TJ}\left(\mathrm{F}_{T J}\right)$ to obtain the line force at the $\mathrm{TJ}$.

The vacancy formation energy at an atomic site $\alpha\left(E_{f}^{\alpha}\right)$ is defined as

$$
E_{f}^{\alpha}=E_{T J}^{\alpha}-E_{T J}+E_{c o h}
$$

here $E_{c o h}$ is the cohesive energy/atom in a perfect fcc lattice and $E_{T J}^{\alpha}$ and $E_{T J}$ are the total energies of the $\mathrm{TJ}$ simulation cell with and without the vacancy, respectively. It is useful to relate the vacancy formation energy in the TJ configuration $\left(E_{f}^{\alpha}\right)$ with that in the bulk $\left(E_{f}^{0}\right)$ to define vacancy binding energy at an atomic site $\alpha$, lying within $4 \mathrm{~nm}$ of the TJ

$$
E_{b}^{\alpha}=E_{f}^{0}=E_{f}^{\alpha}
$$

in order to provide a measure of energy required to move the vacancy from the TJ into the bulk or vice versa. The nudge elastic band method ${ }^{17}$ was employed to study the migration behavior of vacancies along the TJ line. The energy and force minimization criterions used for NEB calculations were 1e-25 and 1e-6, and 8 partitions/replications were used to find the minimum energy path for vacancy migration. The influence of temperature on the activation energy for vacancy migration can be taken into consideration with the help of transition state theory based methods as shown in previous studies ${ }^{41,42}$. Nevertheless, the qualitative findings observed for the intrinsic properties of the TJ and the activation energy would still be consistent at higher temperatures.

1. Valiev, R. Z., Xia, K. \& Langdon, T. G. Processing by severe plastic deformation: an ancient skill adapted for the modern world. Int. J. Mater. Res. 100, 1623-1631 (2009).

2. Meyers, M. A., Mishra, A. \& Benson, D. J. Mechanical properties of nanocrystalline materials. Prog. Mater. Sci. 51, 427-556 (2006).

3. Gleiter, H. Nanostructured materials: basic concepts and microstructure. Acta Mater. 48, 1-29 (2000). 
4. Mullins, W. W. Two-Dimensional Motion of Idealized Grain Boundaries. J. Appl. Phys. 27, 900-904 (1956).

5. Czubayko, U., Sursaeva, V. G., Gottstein, G. \& Shvindlerman, L. S. Influence of triple junctions on grain boundary motion. Acta Mater. 46, 5863-5871 (1998).

6. Gottstein, G., King, A. H. \& Shvindlerman, L. S. The effect of triple-junction drag on grain growth. Acta Mater. 48, 397-403 (2000).

7. Upmanyu, M., Srolovitz, D. J., Shvindlerman, L. S. \& Gottstein, G. Triple Junction Mobility: A Molecular Dynamics Study. Interface Sci. 7, 307-319 (1999).

8. Upmanyu, M., Srolovitz, D. J., Shvindlerman, L. S. \& Gottstein, G. Molecular dynamics simulation of triple junction migration. Acta Mater. 50, 1405-1420 (2002).

9. Gottstein, G., Ma, Y. \& Shvindlerman, L. S. Triple junction motion and grain microstructure evolution. Acta Mater. 53, 1535-1544 (2005).

10. Srinivasan, S. G., Cahn, J. W., Jónsson, H. \& Kalonji, G. Excess energy of grainboundary trijunctions: an atomistic simulation study. Acta Mater. 47, 2821-2829 (1999)

11. King, A. H. The geometric and thermodynamic properties of grain boundary junctions. Interface Sci. 7, 251-271 (1999).

12. Fortier, P., Palumbo, G., Bruce, G. D., Miller, W. A. \& Aust, K. T. Triple line energy determination by scanning tunneling microscopy. Scr. Metall. Mater. 25, 177-182 (1991).

13. Fortier, P., Miller, W. A. \& Aust, K. T. Triple junction and grain boundary character distributions in metallic materials. Acta Mater. 45, 3459-3467 (1997).

14. Schuh, C. A., Kumar, M. \& King, W. E. Universal features of grain boundary networks in FCC materials. J. Mater. Sci. 40, 847-852 (2005).

15. Kremer, R., Narayanan, R., Shekhar, S. \& King, A. H. On the design of controlled tricrystal specimens for the systematic investigation of static grain boundary triple junction properties. J. Mater. Sci. 40, 2795-2802 (2005).

16. Rohrer, G. S. et al. Comparing calculated and measured grain boundary energies in nickel. Acta Mater. 58, 5063-5069 (2010).

17. Henkelman, G., Uberuaga, B. P. \& Jónsson, H. A climbing image nudged elastic band method for finding saddle points and minimum energy paths. J. Chem. Phys. 113, 9901-9904 (2000).

18. Van Swygenhoven, H., Derlet, P. M. \& Frøseth, A. G. Stacking fault energies and slip in nanocrystalline metals. Nat. Mater. 3, 399-403 (2004).

19. Mishin, Y., Farkas, D., Mehl, M. J. \& Papaconstantopoulos, D. A. Interatomic potentials for monoatomic metals from experimental data and ab initio calculations. Phys. Rev. B 59, 3393 (1999)

20. Mishin, Y., Mehl, M. J., Papaconstantopoulos, D. A., Voter, A. F. \& Kress, J. D. Structural stability and lattice defects in copper: Ab initio, tight-binding, and embedded-atom calculations. Phys. Rev. B 63, 224106 (2001).

21. Liu, X. Y., Ercolessi, F. \& Adams, J. B. Aluminium interatomic potential from density functional theory calculations with improved stacking fault energy. Model. Simul. Mater. Sci. Eng. 12, 665 (2004).

22. Shvindlerman, L. S. \& Gottstein, G. Cornerstones of grain structure evolution and stability: Vacancies, boundaries, triple junctions. J. Mater. Sci. 40, 819-839 (2005).

23. Vattré, A. J., Abdolrahim, N., Kolluri, K. \& Demkowicz, M. J. Computational design of patterned interfaces using reduced order models. Sci. Rep. 4 (2014).

24. Zhang, X. \& Misra, A. Superior thermal stability of coherent twin boundaries in nanotwinned metals. Scr. Mater. 66, 860-865 (2012).

25. Costantini, S., Alippi, P., Colombo, L. \& Cleri, F. Triple junctions and elastic stability of polycrystalline silicon. Phys. Rev. B 63, 045302 (2000).

26. Shekhar, S. \& King, A. H. Strain fields and energies of grain boundary triple junctions. Acta Mater. 56, 5728-5736 (2008).

27. Protasova, S. G., Sursaeva, V. G. \& Shvindlerman, L. S. Study of the motion of individual triple junctions in aluminum. Phys. Solid State 45, 1471-1474 (2003).

28. Lehockey, E. M., Brennenstuhl, A. M. \& Thompson, I. On the relationship between grain boundary connectivity, coincident site lattice boundaries, and intergranular stress corrosion cracking. Corros. Sci. 46, 2383-2404 (2004).

29. Gertsman, V. Y. \& Tangri, K. On the relationship between $\sum^{3 \mathrm{n}}$ boundaries meeting at a triple junction. Scr. Metall. Mater. 32, 1649-1652 (1995).

30. Gertsman, V. Y. \& Tangri, K. Computer simulation study of grain boundary and triple junction distributions in microstructures formed by multiple twinning. Acta Metall. Mater. 43, 2317-2324 (1995).
31. Rajagopalan, M., Bhatia, M. A., Tschopp, M. A., Srolovitz, D. J. \& Solanki, K. N. Atomic-scale analysis of liquid-gallium embrittlement of aluminum grain boundaries. Acta Mater. 73, 312-325 (2014).

32. Bhatia, M. A. \& Solanki, K. N. Energetics of vacancy segregation to symmetric tilt grain boundaries in hexagonal closed pack materials. J. Appl. Phys. 114, 244309 (2013).

33. King, A. H. Triple lines in materials science and engineering. Scr. Mater. 62, 889-893 (2010).

34. Mattissen, D., Molodov, D. A., Shvindlerman, L. S. \& Gottstein, G. Drag effect of triple junctions on grain boundary and grain growth kinetics in aluminium. Acta Mater. 53, 2049-2057 (2005).

35. Plimpton, S. Fast parallel algorithms for short-range molecular dynamics. J. Comput. Phys. 117, 1-19 (1995).

36. Pond, R. C. \& Vitek, V. Periodic Grain Boundary Structures in Aluminium. I. A Combined Experimental and Theoretical Investigation of Coincidence Grain Boundary Structure in Aluminium. Proc. R. Soc. Lond. Ser. Math. Phys. Sci. 357, 453-470 (1977)

37. Sutton, A. On the structural unit model of grain boundary structure. Philos. Mag. Lett. 59, 53-59 (1989).

38. Tschopp, M. A. et al. Probing grain boundary sink strength at the nanoscale: Energetics and length scales of vacancy and interstitial absorption by grain boundaries in $\alpha$-Fe. Phys. Rev. B 85, 064108 (2012).

39. Solanki, K. N., Tschopp, M. A., Bhatia, M. A. \& Rhodes, N. R. Atomistic Investigation of the Role of Grain Boundary Structure on Hydrogen Segregation and Embrittlement in $\alpha$-Fe. Metall. Mater. Trans. A 44, 1365-1375 (2013).

40. Wu, M. S., Zhou, K. \& Nazarov, A. A. Crack nucleation at disclinated triple junctions. Phys. Rev. B 76, 134105 (2007).

41. Nguyen, L., Baker, K. \& Warner, D. Atomistic predictions of dislocation nucleation with transition state theory. Phys. Rev. B 84, 024118 (2011).

42. Nguyen, L. \& Warner, D. Improbability of Void Growth in Aluminum via Dislocation Nucleation under Typical Laboratory Conditions. Phys. Rev. Lett. 108, 035501 (2012)

\section{Acknowledgments}

The authors gratefully acknowledge support from the Office of Naval Research under contract N00014111079.

\section{Author contributions}

I.A. and K.N.S. equally contributed to this work. I.A. developed the necessary simulation techniques and performed all the calculations. All the authors discussed the results and wrote the manuscript.

\section{Additional information}

Supplementary information accompanies this paper at http://www.nature.com/ scientificreports

Competing financial interests: The authors declare no competing financial interests.

How to cite this article: Adlakha, I. \& Solanki, K.N. Structural stability and energetics of grain boundary triple junctions in face centered cubic materials. Sci. Rep. 5, 8692; DOI:10.1038/srep08692 (2015)

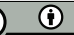

This work is licensed under a Creative Commons Attribution 4.0 International License. The images or other third party material in this article are included in the article's Creative Commons license, unless indicated otherwise in the credit line; if the material is not included under the Creative Commons license, users will need to obtain permission from the license holder in order to reproduce the material. To view a copy of this license, visit http://creativecommons.org/licenses/by/4.0/ 